\title{
Anne-Marie Monluçon et Agathe Salha, Fictions biographiques $\mathrm{XIX}^{\mathrm{e}}-\mathrm{XXI}$ siècles
}

\section{Andrea Manara}

\section{OpenEdition}

\section{Journals}

\section{Edizione digitale}

URL: http://journals.openedition.org/studifrancesi/9389

DOI: 10.4000/studifrancesi.9389

ISSN: 2427-5856

\section{Editore}

Rosenberg \& Sellier

\section{Edizione cartacea}

Data di pubblicazione: 1 juin 2008

Paginazione: 242-244

ISSN: 0039-2944

\section{Notizia bibliografica digitale}

Andrea Manara, «Anne-Marie Monluçon et Agathe Salha, Fictions biographiques $x \mid x^{\mathrm{e}}-x_{x}{ }^{\mathrm{e}}$ siècles», Studi Francesi [Online], 154 (LII | I) | 2008, online dal 30 novembre 2015, consultato il 10 janvier 2021. URL: http://journals.openedition.org/studifrancesi/9389; DOI: https://doi.org/10.4000/studifrancesi.9389

Questo documento è stato generato automaticamente il 10 janvier 2021.

\section{(a) $\odot \Theta$}

Studi Francesi è distribuita con Licenza Creative Commons Attribuzione - Non commerciale - Non opere derivate 4.0 Internazionale. 


\title{
Anne-Marie Monluçon et Agathe Salha, Fictions biographiques XIX $\mathrm{X}^{\mathrm{e}} \mathrm{XXI}$ siècles
}

\author{
Andrea Manara
}

\section{NOTIZIA}

ANNE-MARIE MONLUÇON et AGATHE SALHA, Fictions biographiques XIX ${ }^{e}$-XXI ${ }^{e}$ siècles, Presses Universitaires du Mirail, 2007.

1 «Mon Dieu, que de monde! Qui que tu sois, je te reconnais». Lo strano monito che Karel Capek (citato da M.-O. THIRouin, Les "Récits apocryphes" de Karel Capek, pp. 203-222) rivolge al lettore con la voce del suo personaggio autobiografico esprime bene l'inquietudine di un genere preso fra fiction e documento, letteratura e vita, dove sogno e realtà concorrono a realizzare la possibilità di 'una vita', frammento imperfetto di un sogno ad occhi aperti, staccato dal fondo oscuro e persistente del nulla di un'identità scomparsa. Continua Capek: «nous sommes si égaux, les plus égaux des égaux, égaux dans la possibilité même de la plus extrême différence [...] Plus je lui [mon prochain] serai proche, plus je me comprendrai moi même».

2 Il volume - frutto dell'omonima conferenza che si è svolta all'Università "Stendhal" di Grenoble dall'11 al 14 maggio 2004 (alla quale sono intervenuti, tra gli altri, gli autori: Christian Garcin, Gérard Macé e Jean-Benoît Puech), intende esplorare le forme che la «biographie imaginaire, fictionnelle, d'une personne de la réalité» (M. LAFON, Histoires infâmes, biographies synthétiques, fictions: vies de Jorge Luis Borges, pp. 191-202) ha assunto a partire dal xIX secolo, delimitandone i confini con biografie tradizionali da una parte e romanzo biografico dall'altra. Affinata da un approccio comparatista, l'indagine sulla cronologia individua nell'opera di Marcel Schwob Vies imaginaires (1896) il 'gesto fondatore' e isolato del genere, la cui influenza, benché talvolta sotterranea, si è rivelata decisiva anche al di fuori dei confini francesi. Al contrario, la rinascita 
contemporanea della biografia letteraria si presenta come una «coincidence de multiples publications convergentes» (A.-M. MONLUÇON, A. SALHA, Introduction. Fictions biographiques XIX ${ }^{e}-\mathrm{XXI}^{e}$ siècles: un jeu sérieux?, pp. 7-32), un vero e proprio fenomeno editoriale e teorico in grado, dopo due secoli di ostilità, di attirare l'attenzione della critica letteraria, dove si impone progressivamente la nozione di 'fiction biographique'. Se agli inizi degli anni Ottanta la biografia appariva come un genere reazionario e regressivo, sul finire del decennio essa realizza la propria rinascita e metamorfosi avvalendosi anche dei progressi compiuti dalla scienza storica moderna. Gli storici infatti, in virtù di un intenso processo di revisione critica, rivalutano la biografia facendone il laboratorio di una scrittura inventiva dove la rinnovata soggettività del discorso consente sia l'interesse per gli sconosciuti protagonisti della micro-storia, che un dérapage controllato verso la finzione romanzesca, riconosciuta finalmente come efficace possibilità di ricerca e di inchiesta (A.-E. DEMARTINI, Le retour au genre biographique en histoire: quels renouvellements historiographiques?, pp. 77-89). Probabilmente - ed è ciò che il volume intende dimostrare -, ancor prima del processo avviato dagli storici, la rinascita contemporanea della fiction biographique ha compiuto uno sconvolgimento analogo nel campo della letteratura, dove, maturata una coscienza inquieta e sospettosa nei confronti di rappresentazioni e discorsi (nonché del sapere tout court), ha assimilato tutti i progressi compiuti dal romanzo moderno e concernenti, ad esempio, l'impiego della temporalità o del personaggio. A queste condizioni la fiction biographique moderna identificherà un corpus di testi brevi e spesso frammentari, che inaugurano un ritorno al soggetto e al reale che non riconduce affatto allo status quo ante, poiché riposa su una soggettività assunta: «volontiers ludiques et démystificateurs, [ces textes] visent à restituer la vie d'un personnage réel tout en constituant pour l'écrivain un lieu privilégié de réflexion sur lui-même» (J. ANSELMINI, Fiction biographique moderne et roman historique romantique, la "San Felice" d'Alexandre Dumas, pp. 93-103).

3 Nell'era del sospetto difficoltà insolubili si abbattono come maledizioni sull'esigenza di verità del biografo, il quale, ormai solo e privo di appigli universali, a strapiombo su «l'océan de l'inconnaissable» (D. MADELÉNAT, Le vrai, le faux, le figuré: à propos de David Bellos, "George Perec. Une vie dans les mots" et d'“Antibiotiques", pp. 289-304), vede dissiparsi l'edificio illusorio della biografia totalizzante. "Une scission traverse le personnage et son récit» (J. AUCAGNE, La mise en scène de la relation biographique dans "Le Médecin de Lord Byron" de Paul West, pp. 329-340), e lungo il solco tracciato dall'orbita delle parole si infrange inesorabilmente l'ombra di ogni presenza reale: nell'epoca delle immagini del mondo, il senso della realtà sfuma appiattito sul fascio di figure che ad essa viene sovrapposto, e anche l'uomo, il soggetto, sembra venire travolto nelle dissolvenze, nella proliferazione dei contesti e delle prospettive, dove non esiste verità, né falsità.

4 A queste condizioni, per far fronte a «l'assaut polymorphe de l'erreur» (D. Madelénat), il biografo rivendica allora non tanto l'invenzione, quanto «l'exercice d'une autorité incontestable» (E. Bouju, 'Auctor in fabula/opus a fabula', le double fond de la fiction biographique, pp. 305-315) su testi letterari e documenti storici ricomposti nella forma inedita «d'un patchwork [...] une écriture seconde qui se dénonce comme telle» (J. Aucagne), frutto di «un art consommé du raccord et de l'agencement des parties» (E. STEAD, "Des graines pleines d'une essence violente" dans "Vies imaginaires": Marcel Schwob et les Élisabéthains, pp. 157-175) che, attraversando tutto un sapere accumulato di materiali 
d'archivio, interpretazioni e rappresentazioni già costituite in discorsi, si decentra dall'illusorio manicheismo del vero contrapposto al falso: «comme la coquille, [qui] se meut autour d'un axe imaginaire» (M. MACÉ, Un 'Total fabuleux': biographies intellectuelles et mobilisation de la fiction, pp. 259-274), l'arte si avvolge sullo strano ritmo del passo a due con cui un ordine immaginario riorganizza l'informazione disponibile, restituendo una visione parziale, un succedaneo indiretto, fittizio e sostituibile, di un'esistenza trasfigurata nella costruzione testuale.

5 Al di là dell'aspetto estetico, tuttavia, se ormai «l'homme est trop grand pour n'avoir qu'un seul visage» (Capek citato da M.-O. Thirouin) e tutta la realtà viene pensata come «fiction potentielle laissée en attente de témoin-transcripteur» (A. GEFEN, Soi-même comme un autre: présupposés et significations du recours à la fiction biographique dans la littérature française contemporaine, pp. 55-75), la fiction biographique non si rassegna al nichilismo epistemologico. Volendo fare «de l'acte biographique un voyage, une enquête, plutôt qu'un dépôt ou un bilan» (M. SHERINGAM, Sartre et la compréhension du vécu, pp. 275-287), essa riorganizza le sue fonti in temibili enunciati performativi, che non si relazionano più al reale unicamente nel senso di mimesis, ma piuttosto in termini di 'intervention' (A.-M. Monluçon, A. Salha), sul modo del riscatto o della critica dissacrante e demistificatoria, dello smascheramento di realtà occultate o della configurazione narrativa di processi di responsabilità. Sarebbe riduttivo vedere nella fiction biographique un mero prodotto post-moderno votato alla confusione del vero e del falso, alla trasgressione dei generi, ad una sterile critica delle rappresentazioni e alla decostruzione ludica e nichilista. Il panorama offerto, aperto sia sul piano storico che su quello geografico e linguistico, mette a fuoco tanto una categoria di testi avveduti e informati di una coscienza antipositivista, quanto l'«acte de pure prétention, un simple geste [qui] pourtant s'avère parfois efficace» (A. JEFFERSON, Imposture et croyance dans les "Vies minuscules" de Pierre Micron, pp. 247-256) e forte di una ritrovata fiducia nelle potenzialità euristiche della letteratura, di una scrittura in grado di «concilier l'héritage de la modernité et sa critique post-moderne» (A.-M. Monluçon, A. Salha) in vista di un rinnovamento della storia sociale.

6 A tal punto informata dei propri mezzi, la fiction esibisce la relazione biografica non solo come lavoro di lettura, ma anche come «échange d'altérité [...] une réponse à l'impuissance du biographe à ressembler le composite dans une figure cohérente, comme à la menace de morcellement et de frustration qui pèse sur lui-même» (J. Aucagne). Nutrendosi di un reale già rifranto e disperso in discorsi eterogenei, l'arte del biografo - «ce creusement de soi par la figure de l'autre» (D. Viart) - misura l'estensione di un gesto ambivalente che, componendo astrazioni immaginarie, si completa incrociando l'inquietudine di una ricerca personale: «d'homme, d'artiste, d'écrivain» (B. BоST, L'euvre dramatique de Denis Guénoun: portraits de grands hommes et figure d'auteur, pp. 145-154). La grande espansione del genere negli anni '80 riflette un più generico affiorare dell'individuo (riscontrabile anche nel proliferare del genere contiguo dell'autobiografia) dallo stato di latenza cui negli anni delle grandi speranze collettive era stato confinato. E non lo ritroviamo dove sarebbe legittimo aspettarsi, ma lo cogliamo, per così dire, in flagrante, dove è sempre rimasto: un enigmatico détour par autrui, su traiettorie che ricordano quelle del vampiro «qui renaît sans cesse et traverse la mort en se nourrissant de la vie d'autrui» (J. Aucagne), gli consente di sporgere dal testo letterario mantenendosi al riparo dalle insidie delle convenzioni autobiografiche. L'io e l'altro si confondono sulle silhouettes di personaggi reinventati «à milles lieues de 
la biographie traditionnelle» (Pontalis, citato nell'introduzione), cancellando il confine fra biografia e autobiografia dallo sfondo di un solo genere 'bipolare', che li stringe in una relazione paradossale in cui le scelte relative al secondo sono innanzitutto rivelatrici del primo: «servitude et indépendance, entre la vie pour soi et la vie pour l'autre - les deux pôles étant à chaque fois tragiquement indissociables [...], pris dans cette dialectique inextricable: créer, et être créé» (J. Aucagne). Al di là del riferimento alla deflagrazione dei generi letterari caratteristica della nostra epoca e del fenomeno, indissociabile dall'idea di letteratura, della proiezione dell'autore nell'universo che crea nelle sue fiction, il ricorso esplicito alla biografia come a una forma legittima della scrittura di sé riflette un tratto fondamentale della cultura letteraria contemporanea: «le besoin de penser soi-même comme un autre[...] de se voir dans autrui et de retrouver autrui en soi» (A. Gefen). Nel quadro di una «contestation 'disloquante' [...] de la vie comme forme relativement unifiée» (J.-F. LOUETTE, Henri Michaux: 'Une vie de chien' (Mes propriétés), pp. 343-357), il riconoscimento di sé, ormai privo di centro, è un processo trasmigratorio che gira a vuoto, un miraggio costantemente rinegoziato, intersoggettivo e intertestuale, per cui «le recueil biographique fournit alors une matrice exemplaire de l'entreprise autobiographique» (L. DEMENZE, Les illustres et les minuscules, Pierre Michon, lecteur de Plutarque, pp. 235-246).

Il xx secolo che si è appena concluso sembra aver relegato il segreto dell'identità personale in un incavo nascosto dalle imposture della coscienza e dalle dissimulazioni dell'inconscio, mettendo senz'altro in discussione la coincidenza di autore, narratore e personaggio su cu fa leva la nozione di 'patto autobiografico' descritta da P. Lejeune. Una poetica del sospetto impedisce ormai all' 'io' che sottende la scrittura autobiografica di colmare la distanza che separa soggetto dell'enunciato e soggetto dell'enunciazione, per cui questa figura secondaria, sintesi di esperienze multiple, nasconde sempre in realtà «une troisième personne occultée, et, en ce sens, toute autobiographie est par définition indirecte» (P. Lejeune, citato in L. Demenze). Il narratore omodiegetico instaura una relazione ibrida col suo creatore, «ni, bien sûr, simple double de l'auteur, ni totalement libre de toute trace de relation mimétique» (R. Kahn), spia di una tensione costantemente ricominciata verso un reale intimo non assegnabile, che tuttavia non si perde nell'illusione di una coincidenza del dire col suo oggetto. Non solo, ma un'insistenza marcata sui criteri di coincidenza - in particolare quando ci si avvale anche di autoritratti fotografici, come nel caso di W.G. Sebald descritto da R. Kahn - «produit moins une insertion de la personne réelle dans un récit en grande partie fictionnel, qu'une fictionnalisation de la persone même de l'écrivain transformé en personnage» (R. Kahn).

8 Se, come dice Valéry, «vivre c'est se transformer dans l'incomplet» (M. Macé), superati l'illusione di un racconto totalizzante e lo scoglio di uno scetticismo senza sbocchi, si accede ad una dimensione primordiale di «possibilités insoupçonnées», dove «[toute vie] aurait pu être autre [...] somme de toute les vies réalisées et révées» (M.-O. Thirouin). Ridotto in pezzi e preso, voltandosi, nella rotazione continua di un gesto irresistibile che intreccia il vero e il falso sulla spirale dell'immaginario, lo scrittore è il sogno di qualcun altro, «ver à soie [...] qui tisse autour de soi ce cocon presque infini qu'est son texte» (R. Kahn).

«Mon Dieu, que de monde! Qui que tu sois, je te reconnais». 